\title{
Fatores associados à Sarcopenia em idosos da comunidade
}

\author{
Factors associated with Sarcopenia in Elderly Community \\ Factores asociados con Sarcopenia en ancianos de la comunidad \\ Juliana Duarte Nunes ${ }^{1}$, Juliana de Fátima Zacarin², Sofia Cristina lost Pavarini³, Marisa Silvana Zazzetta4, \\ Ariene Angelini dos Santos Orlandi ${ }^{5}$, Fabiana de Souza Orlandi ${ }^{6}$
}

RESUMO | O objetivo deste estudo foi identificar os fatores associados à sarcopenia em idosos da comunidade. Tratou-se de um estudo correlacional, de corte transversal e abordagem quantitativa, realizado com 234 idosos da comunidade. Para avaliar a sarcopenia foram utilizados os critérios do European working group on sarcopenia in older people (EWGSOP2) e para coleta de dados foram utilizados: questionário sociodemográfico e de saúde para caracterização dos idosos; escala de depressão geriátrica, Addenbrooke's cognitive examinationrevised, international physical activity questionnaire, versão longa e o medical outcomes study 36-item short-form health survey. Houve predominância de pessoas do sexo feminino ( $n=151 ; 68,30 \%)$, de cor branca ( $n=163 ; 73,80 \%)$, com sobrepeso, segundo o Índice de Massa Corporal (IMC) ( $n=124 ; 56,10 \%)$ e $24,90 \% \quad(n=55)$ dos participantes apresentaram sarcopenia, segundo os critérios do consenso europeu. Se associaram à sarcopenia os seguintes fatores: idade $(\mathrm{OR}=1,06 ; \quad \mathrm{p}=0,010), \quad \mathrm{M} \mathrm{MC}<22 \mathrm{~kg} / \mathrm{m}^{2}$ $(O R=5,82 ; \quad p=0,003)$, ser insuficientemente ativo fisicamente $(O R=3,29 ; p=0,002)$ e presença de quedas $(\mathrm{OR}=2,20 ; \mathrm{p}=0,033)$. Os idosos com idade avançada, com baixo peso, insuficientemente ativos fisicamente e que sofreram quedas têm mais chance de apresentar sarcopenia.

Descritores I Sarcopenia; Idoso; Envelhecimento
ABSTRACT I Our study aimed to identify the factors associated with sarcopenia in older adults in the community. This is a correlational, cross-sectional study with a quantitative approach, conducted with 234 older people. We used the criteria of the European Working Group on Sarcopenia in Older People (EWGSOP2) to assess sarcopenia and collected the following data: sociodemographic and health questionnaire to characterize older adults; geriatric depression scale, Addenbrooke's cognitive exam - revised, international physical activity questionnaire long version and the medical outcomes study 36-item short-form health survey. Most participants were women ( $n=151 ; 68.30 \%$ ), white ( $n=163 ; 73.80 \%$ ), overweight, according to the body mass index - BMI $(n=124 ; 56.10 \%)$ and $24.90 \%(n=55)$ of them presented sarcopenia, according to the criteria of the European Consensus. The following factors were associated with sarcopenia: age $(O R=1.06$; $p=0.010)$, $\mathrm{BMI}<22 \mathrm{~kg} / \mathrm{m}^{2}(\mathrm{OR}=5.82 ; \mathrm{p}=0.003)$, being insufficiently physically active $(O R=3.29 ; p=0.002)$ and reports of falls (OR=2.20; $p=0.033)$. Older people, with low weight, according to BMI, insufficiently physically active and that suffered falls are more likely to have sarcopenia.

Keywords | Sarcopenia; Aged; Aging.

RESUMEN I El objetivo de este estudio fue identificar los factores asociados a la sarcopenia en ancianos de la comunidad Se trata de un estudio correlacional, transversal,

\footnotetext{
A pesquisa foi realizada na cidade de São Carlos (SP).

Universidade Federal de São Carlos (UFSCar) - São Carlos (SP), Brasil. E-mail: forlandi@ufscar.br. ORCID-0000-0001-7678-038X 2Universidade Federal de São Carlos (UFSCar) - São Carlos (SP), Brasil. E-mail: forlandi@ufscar.br. ORCID-0000-0001-5053-0868. ¿Universidade Federal de São Carlos (UFSCar) - São Carlos (SP), Brasil. E-mail: forlandi@ufscar.br ORCID-0000-0001-9359-8600 4Universidade Federal de São Carlos (UFSCar) - São Carlos (SP), Brasil. E-mail: forlandi@ufscar.br. ORCID-0000-0001-6544-767X 5Universidade Federal de São Carlos (UFSCar) - São Carlos (SP), Brasil. E-mail: forlandi@ufscar.br. ORCID-0000-0002-3112-495X ${ }^{6}$ Universidade Federal de São Carlos (UFSCar) - São Carlos (SP), Brasil. E-mail: forlandi@ufscar.br. ORCID-0000-0002-5714-6890
} 
con abordaje cuantitativo, realizado con 234 ancianos de la comunidad Para evaluar la sarcopenia se utilizaron los criterios del European working group on sarcopenia in older people (EWGSOP2), y para la recolección de datos se utilizaron: cuestionario sociodemográfico y de salud para caracterizar a Ios ancianos; escala de depresión geriátrica, Addenbrooke's cognitive examination-revised, international physical activity questionnaire, versión larga, y el medical outcomes study 36item short-form health survey. Hubo un predominio de mujeres ( $n=151 ; 68,30 \%$ ), blancas ( $n=163$; el $73,80 \%$ ), con sobrepeso, de acuerdo con el Índice de Masa Corporal (IMC) ( $n=124 ; 56,10 \%)$ y 24,90\% ( $n=55)$ de los participantes presentaron la sarcopenia, de acuerdo a los criterios del consenso europeo. Los siguientes factores se asociaron a la sarcopenia: edad ( $O R=1,06 ; p=0,010)$, IMC $<22 \mathrm{~kg} / \mathrm{m}^{2}(\mathrm{OR}=5,82 ; \mathrm{p}=0,003)$, estar insuficientemente activo físicamente $(O R=3,29 ; p=0,002)$ y la presencia de caídas $(\mathrm{OR}=2,20 ; \mathrm{p}=0,033)$. Los ancianos con edad avanzada, con bajo peso, insuficientemente activos físicamente y que han sufrido caídas tienen más probabilidades de padecer sarcopenia. Palabras clave I Sarcopenia; Ancianos; Envejecimiento.

\section{INTRODUÇÃO}

A sarcopenia é uma doença muscular que pode ser definida com uma perda involuntária de massa muscular esquelética e força relacionada à idade ${ }^{1}$. Em 2010, o European working group on sarcopenia in olderpeople (EWGSOP) publicou um consenso com os seguintes parâmetros mensuráveis para o diagnóstico de sarcopenia: massa apendicular esquelética, força muscular e desempenho físico ${ }^{1-4}$. Este consenso foi amplamente utilizado e em 2018 foi atualizado. Com as novas recomendações do EWGSOP 2 a baixa força muscular passou a ser o parâmetro primário e o diagnóstico é confirmado pela presença de baixa quantidade ou qualidade muscular. A sarcopenia é classificada como grave quando se soma à baixa performance física ${ }^{2}$.

Uma meta-análise incluiu 35 estudos com idosos da comunidade, com idade de 60 anos ou mais, no qual a sarcopenia foi avaliada segundo os critérios do EWGSOP ${ }^{5}$. Os autores desta meta-análise encontraram uma prevalência total de $10 \%$ (95\% CI=8-12\%) para homens e $10 \%$ (95\% CI=8-13\%) para as mulheres ${ }^{5}$. Estes números destacam a necessidade de tratar a sarcopenia como um problema de saúde pública, já que em hospitais e nas instituições de longa permanência, tanto a prevalência como a incidência são susceptíveis a serem significativamente mais altas ${ }^{6,7}$.

Um estudo longitudinal realizado na Toscana, Itália, com 538 participantes da comunidade, objetivou verificar os valores preditivos da sarcopenia em relação a desenvolvimento de futuras deficiências, hospitalização e mortalidade ${ }^{8}$. Os autores, utilizando-se dos critérios de sarcopenia estabelecidos pelo EWGSOP, identificaram que $10,2 \%$ dos idosos estavam sarcopênicos. Neste estudo foi verificado que $22 \%$ dos idosos sarcopênicos apresentaram deficiências nas atividades instrumentais de vida diária, além de que idosos sarcopênicos apresentaram risco significativamente maior de desenvolver essas deficiências quando comparados com os idosos não-sarcopênicos. Além disso, a hospitalização, as incapacidades e a mortalidade ${ }^{8}$ se associaram conjuntamente na predição de sarcopenia.

É de extrema importância o cuidado para as pessoas com sarcopenia, dado que tal condição provoca altos encargos pessoais, sociais e econômicos, quando não tratada ${ }^{8}$. De forma geral, a sarcopenia aumenta o risco de quedas e fraturas ${ }^{9,10} \mathrm{e}$ prejudica a capacidade de realizar atividades de vida diária ${ }^{11}$. É associada a doenças cardíacas ${ }^{12}$, doenças respiratórias ${ }^{13} \mathrm{e}$ prejuízo cognitivo ${ }^{14}$. Ela também contribui para a diminuição da qualidade de vida ${ }^{15-17}$, perda de independência, necessidade de cuidados de longo prazo ${ }^{18-20}$ e morte ${ }^{21}$.

Em termos financeiros, a sarcopenia é cara para os sistemas de saúde, pois aumenta o risco de hospitalização e todos os custos decorrentes de uma dependência maior de cuidado ${ }^{22}$. Pesquisas internacionais mostraram que pessoas idosas sarcopênicas possuem um custo de hospitalização de duas a cinco vezes maior, em relação àquelas que não possuem sarcopenia ${ }^{22-25}$, sendo assim, é primordial que se desenvolvam ações preventivas, atuando nos fatores de risco que predispõem à sarcopenia.

Frente ao exposto, o objetivo dessa pesquisa foi identificar os fatores associados à sarcopenia em pessoas idosas da comunidade.

\section{METODOLOGIA}

Trata-se de um estudo correlacional, de corte transversal e abordagem quantitativa, realizado com 234 idosos cadastrados em unidades de saúde da família (USF) de um município do interior do estado de São Paulo, Brasil. Foram entrevistadas as pessoas idosas que atenderam os 
seguintes critérios de inclusão: estar cadastrado em uma USF e ter 60 anos de idade ou mais. Os critérios de exclusão adotados foram: possuir déficit de audição e/ou de visão grave que impossibilitassem o entendimento e responder aos instrumentos. Para a coleta de dados foram utilizados: questionário sociodemográfico e de saúde, elaborado pela equipe do estudo, para caracterização dos participantes; escala de depressão geriátrica-15 (GDS) ${ }^{26}$; Addenbrooke's cognitive examination-revised (ACE-R $)^{27}$; questionário internacional de atividade física (IPAQ) ${ }^{28}$; medical outcomes study e 36-item short-form health survey (SF-36) ${ }^{29}$. As entrevistas para a coleta de dados foram previamente agendadas e realizadas no domicílio dos participantes.

Para diagnosticar a sarcopenia foram adotados os critérios recomendados pelo EWGSOP2, para isso avaliou-se a força muscular, confirmou-se a sarcopenia pela massa muscular e determinou-se a gravidade pela performance física. Para avaliar a evidência da sarcopenia nos participantes foi aferida a força de preensão palmar, segundo o critério estabelecido pelo estudo saúde, bem-estar e envelhecimento $(\mathrm{SABE})$ que utiliza nota de corte $<30 \mathrm{Kg}$ para homens e $<20 \mathrm{Kg}$ para mulheres. Para confirmar a sarcopenia pela deteç̧ão de baixa quantidade e qualidade muscular, foi realizado o dual-energy $x$-ray absortiometry (DXA), no Departamento de Fisioterapia da Universidade Federal de São Carlos (UFSCar). Todos os participantes que responderam aos questionários foram submetidos ao exame de densitometria óssea. As notas de corte consideradas foram as sugeridas pelo estudo $\mathrm{SABE}$ sendo $6,37 \mathrm{~m}^{2} / \mathrm{kg}$ para mulheres e $8,90 \mathrm{~m}^{2} / \mathrm{kg}$ para os homens $\mathrm{s}^{30,31}$. Para determinar a gravidade da sarcopenia foi realizado o teste de velocidade da marcha, em ambiente preparado e controlado, com nota de corte menor de $0,8 \mathrm{~m} / \mathrm{s}$ para ambos os sexos ${ }^{1,2}$

Para a definição do tamanho da amostra consideraramse as pontuações dos instrumentos de coleta de dados. Por hipótese assumida, haveria correlação entre esses instrumentos, portanto, o cálculo do tamanho da amostra baseou-se no coeficiente de correlação de Pearson, sendo o coeficiente arbitrado de 0,20 a 0,50 , com intervalo de 0,05 , com poder do teste de $80 \%(\beta=0,20)$ e a confiança de $95 \%$. Para um r=0,20 (que representa uma correlação baixa), com base nos estudos internacionais ${ }^{32}$, o tamanho amostral seria de 194 sujeitos.

Os dados coletados foram transportados para uma tabela em formato Excel 2007 e foi utilizado o programa estatístico statistical package for the social sciences (SPSS) versão 20.0 para as análises. Foi realizada uma análise descritiva das variáveis categóricas e contínuas.
Para analisar os efeitos das variáveis independentes sobre a sarcopenia foi realizada análise de regressão logística binária multivariada. As variáveis independentes que se associaram à sarcopenia nas pessoas idosas da comunidade, com $\mathrm{p}$-valor $\leq 0,20$ entraram no modelo, excetuando-se as colineares. Por meio do método de seleção forward foram mantidas no modelo final as variáveis independentes que conjuntamente obtiveram p-valor $\leq 0,05$.

As variáveis numéricas (independentes) incluídas de forma contínua foram: idade (em anos), escolaridade (em anos), renda per capita mensal (em reais), número de medicamentos (em unidades); número de doenças associadas (em unidades); índice de massa corporal (em $\mathrm{kg} / \mathrm{m}^{2}$ ); número de quedas (em unidades). Enquanto as variáveis qualitativas (independentes) receberam valores 0 ou 1 para identificar suas categorias. $O$ nível de significância adotado para os testes estatísticos foi de $5 \%(\mathrm{p}<0.05)$.

\section{RESULTADOS}

Das 234 pessoas idosas participantes do estudo 68,30\% ( $n=151)$ eram do sexo feminino, com predominância de pessoas de cor branca $(73,80 \%)$ e casadas $(61,10 \%)$. Observou-se que 56,10\% apresentaram IMC $>27 \mathrm{Kg} /$ $\mathrm{m}^{2}, 24,90 \%(\mathrm{n}=55)$ apresentaram sarcopenia, segundo os critérios do consenso europeu e 33,00\% tiveram pelo menos uma queda nos últimos doze meses. A maioria dos idosos possuíam média de 69,37 anos de idade, 5,44 anos de estudo e uma renda média per capta de $1.200,25$ reais. Destaca-se também que a média de medicamentos diários foi de 3,65 e 4,44 doenças por participante (Tabela 1).

Tabela 1. Distribuição da caracterização sociodemográfica e de saúde dos idosos participantes (n=234) - São Carlos (2019)

\begin{tabular}{|c|c|c|}
\hline Variáveis & n (\%) & $\begin{array}{c}\text { Média } \\
( \pm \text { desvio padrão) }\end{array}$ \\
\hline \multicolumn{3}{|l|}{ Gênero } \\
\hline Feminino & $151(68,30)$ & \\
\hline Masculino & $70(31,70)$ & \\
\hline \multicolumn{3}{|l|}{ Estado Civil } \\
\hline Casado & $135(61,10)$ & \\
\hline Viúvo & $53(24,00)$ & \\
\hline Divorciado & $18(8,10)$ & \\
\hline Solteiro & $15(6,80)$ & \\
\hline
\end{tabular}

(continues) 


\begin{tabular}{|c|c|c|}
\hline Variáveis & $n(\%)$ & $\begin{array}{c}\text { Média } \\
( \pm \text { desvio padrão) }\end{array}$ \\
\hline \multicolumn{3}{|l|}{ Etnia } \\
\hline Branca & $163(73,80)$ & \\
\hline Negra & $28(12,70)$ & \\
\hline Parda & $27(12,20)$ & \\
\hline Amarela & $3(1,40)$ & \\
\hline \multicolumn{3}{|l|}{ IMC $\left(\mathrm{Kg} / \mathrm{m}^{2}\right)$} \\
\hline$<22$ & $17(7,70)$ & \\
\hline 22 a 27 & $80(36,20)$ & \\
\hline$>27$ & $124(56,10)$ & \\
\hline Idade & & $69,37(7,03)$ \\
\hline Escolaridade & & $5,44(4,37)$ \\
\hline Renda per capita & & $1200,25(1352,20)$ \\
\hline $\begin{array}{l}\text { Número de } \\
\text { medicamentos }\end{array}$ & & $3,65(4,07)$ \\
\hline Número de doenças & & $4,44(3,35)$ \\
\hline \multicolumn{3}{|l|}{ Sarcopenia } \\
\hline Não & $166(75,10)$ & \\
\hline Sim & $55(24,90)$ & \\
\hline \multicolumn{3}{|l|}{ Quedas } \\
\hline Não & $148(67,00)$ & \\
\hline Sim & $73(33,00)$ & \\
\hline
\end{tabular}

A Tabela 2 apresenta os resultados da análise de regressão logística univariada. Para verificar os fatores associados à sarcopenia nos idosos, as variáveis independentes foram incluídas separadamente, apresentadas no modelo abaixo (tabela 2). É possível observar que as variáveis que associaram-se à sarcopenia nos idosos foram a idade, número de medicamentos em uso, IMC $<22 \mathrm{Kg} / \mathrm{m}^{2}$, circunferência da panturrilha, ser insuficientemente ativo fisicamente, bom desempenho cognitivo, ter melhor pontuação no GDS, capacidade funcional e ter bom estado geral de saúde.

Tabela 2. Análise de regressão logística univariada para sarcopenia $(n=234)$ - São Carlos, 2019

\begin{tabular}{|c|c|c|c|c|}
\hline Variável & Categorias & p-valor & $\mathrm{OR}^{*}$ & IC 95\%* \\
\hline Idade & Variável contínua & $<0,001$ & 1,108 & $1,059-1,158$ \\
\hline Sexo & $\begin{array}{l}\text { Homem } \\
\text { Mulher }\end{array}$ & $\begin{array}{r}\text { Ref. } \\
0,127\end{array}$ & $\begin{array}{r}-- \\
1,639\end{array}$ & $0,868-3,094$ \\
\hline Quedas & $\begin{array}{l}\text { Sim } \\
\text { Não }\end{array}$ & $\begin{array}{r}0,055 \\
\text { Ref. }\end{array}$ & $\begin{array}{r}1,849 \\
--\end{array}$ & $\begin{array}{r}0,986-3,466 \\
--\end{array}$ \\
\hline $\begin{array}{l}\text { Número de } \\
\text { medicamentos }\end{array}$ & Variável contínua & 0,008 & 1,104 & $1,026-1,187$ \\
\hline $\begin{array}{l}\text { Número de } \\
\text { doenças } \\
\text { associadas }\end{array}$ & Variável contínua & 0,235 & 1,055 & $0,966-1,153$ \\
\hline
\end{tabular}

\begin{tabular}{|c|c|c|c|c|}
\hline Variável & Categorias & p-valor & $\mathrm{OR}^{*}$ & IC 95\%* \\
\hline $\begin{array}{l}\text { Recebe } \\
\text { apoio social } \\
\text { emocional }\end{array}$ & $\begin{array}{l}\text { Sim } \\
\text { Não }\end{array}$ & $\begin{array}{r}\text { Ref. } \\
0,055\end{array}$ & 1,888 & $0,987-3,610$ \\
\hline $\begin{array}{l}\text { Recebe apoio } \\
\text { social material }\end{array}$ & $\begin{array}{l}\text { Sim } \\
\text { Não }\end{array}$ & $\begin{array}{r}\text { Ref. } \\
0,226\end{array}$ & $\begin{array}{r}-- \\
1,461\end{array}$ & $0,791-2,698$ \\
\hline $\begin{array}{l}\text { Índice de } \\
\text { Massa Corporal }\end{array}$ & $\begin{array}{l}<22 \mathrm{~kg} / \mathrm{m}^{2} \\
\geq 22 \mathrm{~kg} / \mathrm{m}^{2}\end{array}$ & $\begin{array}{r}<0,001 \\
\text { Ref. }\end{array}$ & $\begin{array}{r}6,625 \\
--\end{array}$ & $\begin{array}{r}2,320-18,918 \\
--\end{array}$ \\
\hline $\begin{array}{l}\text { Circunferência } \\
\text { da Panturrilha }\end{array}$ & Variável contínua & $<0,001$ & 0,791 & $0,717-0,872$ \\
\hline $\begin{array}{l}\text { Nível de } \\
\text { Atividade } \\
\text { Física }\end{array}$ & $\begin{array}{l}\text { Ativo } \\
\text { Insuficientemente } \\
\text { ativo }\end{array}$ & $\begin{array}{r}\text { Ref. } \\
<0,001\end{array}$ & 4,169 & $2,117-8,209$ \\
\hline ACE-R total & Variável contínua & $<0,001$ & 0,964 & $0,948-0,980$ \\
\hline GDS total & Variável contínua & 0,001 & 1,165 & $1,063-1,278$ \\
\hline $\begin{array}{l}\text { Capacidade } \\
\text { Funcional** }\end{array}$ & Variável contínua & $<0,001$ & 0,977 & $0,966-0,987$ \\
\hline $\begin{array}{l}\text { Estado Geral } \\
\text { de Saúde** }\end{array}$ & Variável contínua & $<0,001$ & 0,972 & $0,958-0,987$ \\
\hline
\end{tabular}

As variáveis que conjuntamente associaram-se à sarcopenia nos idosos avaliados estão no modelo apresentado na tabela 3, em que é possível verificar que idade $(\mathrm{OR}=1,066), \mathrm{IMC}<22 \mathrm{~kg} / \mathrm{m}^{2}(\mathrm{OR}=5,828)$, ser insuficientemente ativo fisicamente $(\mathrm{OR}=3,298)$ e ter sofrido quedas o ano anterior à pesquisa $(\mathrm{OR}=2,201)$ foram os fatores associados à sarcopenia. É possível verificar no modelo, que a cada ano a mais de idade a chance de se ter sarcopenia aumenta em 1.066 vezes, além de que a chance de apresentar sarcopenia aumenta em 5.828 vezes, se desnutrido ou baixo peso, segundo IMC, em 3.298 vezes se insuficientemente ativo e em 2.201vezes se sofreu queda no ano anterior à pesquisa.

Tabela 3. Análise de regressão logística multivariada para sarcopenia $(n=234)$ - São Carlos, SP, (2018)

\begin{tabular}{|c|c|c|c|}
\hline $\begin{array}{c}\text { Variáveis } \\
\text { Selecionadas }\end{array}$ & p-valor & O.R.* & IC 95\% O.R.* \\
\hline Idade (anos) & 0,010 & 1,066 & $1,015-1,120$ \\
\hline $\begin{array}{l}\text { Índice de Massa } \\
\text { Corporal }\end{array}$ & 0,003 & 5,828 & $1,810-18,765$ \\
\hline $\begin{array}{l}\text { Nível de Atividade } \\
\text { Física }\end{array}$ & 0,002 & 3,298 & $1,523-7,143$ \\
\hline Quedas & 0,033 & 2,201 & $1,064-4,551$ \\
\hline Sexo & 0,128 & 1,766 & $0,848-3,677$ \\
\hline
\end{tabular}

*OR (Odds Ratio): Razão de chances para sarcopenia; IC 95\%: Intervalo de 95\% de confiança. Critério Forward de seleção de variáveis. Categorias: idade (variável contínua), IMC $\left(<22 \mathrm{Kg} / \mathrm{m}^{2}\right)$, Nível de Atividade Física (Insuficientemente ativo), Quedas (sim/ocorreu), Sexo (mulher).

\section{DISCUSSÃO}

Neste estudo houve predominância do sexo feminino, de etnia branca, casados, IMC $>27 \mathrm{~kg} / \mathrm{m}^{2}$ e $24,90 \%$ apresentaram sarcopenia, segundo os critérios do EWGSOP 2, corroborando com os achados da literatura 
científica. Os fatores associados de sarcopenia nos idosos avaliados neste estudo foram: idade $(\mathrm{OR}=1,066)$, IMC $<22 \mathrm{~kg} / \mathrm{m}^{2}(\mathrm{OR}=5,828)$, ser insuficientemente ativo fisicamente $(\mathrm{OR}=3,298)$ e ter sofrido quedas o ano anterior à pesquisa $(\mathrm{OR}=2,201)^{33-36}$.

Petermann-Rocha et al. avaliaram os fatores de risco associados à sarcopenia em 396.283 participantes no $U K$ biobank baseline clinic e descobriram que a idade maior que 65 anos foi associada com a sarcopenia. Além disso, pessoas abaixo do peso ideal apresentaram oito vezes mais chances de apresentar essa condição (OR: 7,60 [95\% CI: 6,08-9,50]) $)^{37}$.

Um estudo desenvolvido na Malásia, com o objetivo de determinar a prevalência de sarcopenia e seus fatores associados entre idosos com diabetes mellitus (DM), usuários da atenção primária à saúde, verificou a proporção de $28,5 \%$ de sarcopenia entre os 506 participantes. Dentre os fatores associados citaram: aqueles com idade $\geq 70$ anos $(\beta=0,73 ; \mathrm{OR}=2,07$; IC 95\%=1,24, 3,48; $p=0,006)$, sexo masculino $(\beta=0,61 ; \mathrm{OR}=1,84$; IC $95 \%=1,12,3,02$; $\mathrm{p}=0,017), \mathrm{com} \geq 10$ anos de duração do diabetes $(\beta=0,62$; $\mathrm{OR}=1,85 ; \mathrm{IC} 95 \%=1,11,3,09 ; \mathrm{p}=0,018)$, uso de menos de cinco medicamentos $(\beta=0,68 ; \mathrm{OR}=1,98$; IC $95 \%=1,17$, $3,36 ; \mathrm{p}=0,011)$, baixo índice de massa corporal (IMC) $(\beta=-2,43 ; \mathrm{OR}=0,09 ; \mathrm{IC} 95 \%=0,05,0,17 ; \mathrm{p}<0,001)$ e envolvimento em atividades físicas leves $(\beta=0,77$; $\mathrm{OR}=2,15 ; \mathrm{IC} 95 \%=1,07,4,35 ; \mathrm{p}=0,032)$ e moderadas $(\beta=0,80 ; \text { OR=2,23; IC 95\%=1,07, 4,66; } p=0,033)^{35}$.

Não somente, em uma pesquisa realizada em SalvadorBrasil, com o objetivo de verificar a prevalência e os fatores associados à sarcopenia em idosos institucionalizados ${ }^{36}$, encontrou-se uma maior prevalência de sarcopenia (23\%) entre pessoas idosas com magreza $(\mathrm{OR}=1,28$; IC 95\%: 1,14-1,43) em relação aos idosos eutróficos.

Tramontano et al. avaliaram a prevalência da sarcopenia e seus fatores associados em 222 indivíduos com idade superior a 65 anos na Cordilheira dos Andes, e encontraram que a idade avançada e a baixa prática de atividade física foram fatores preditores de sarcopenia ${ }^{38}$.

Com relação à prática de atividade física, diversos estudos disponíveis na literatura científica citam que a inatividade física contribui para o desenvolvimento da sarcopenia ${ }^{39,40}$. Em uma pesquisa longitudinal realizada na Inglaterra em um período de oito anos, a qual analisou os fatores de risco para sarcopenia em 1.564 participantes, os autores encontraram a associação inversa entre atividade física e risco de sarcopenia ${ }^{41}$.

O presente estudo demonstrou ainda que a sarcopenia foi correlacionada a quedas. Diversos estudos indicam que indivíduos idosos sarcopênicos apresentam um risco significativamente elevado de sofrerem quedas em comparação a idosos não sarcopênicos ${ }^{42-44}$. Em uma investigação realizada na Espanha com 235 idosas, Aibar-Almazán et al. encontraram a associação entre a sarcopenia e quedas ${ }^{45}$.

Não somente, Lim et al.investigaram a associação entre a sarcopenia e quedas em 147 pacientes idosos de diversos hospitais da Coreia do $\mathrm{Sul}^{46} \mathrm{com}$ idade superior a 65 anos, com fratura de quadril. Como resultado, os autores encontraram correlação significativa entre a sarcopenia e queda $(r=0,222, p=0,007)$. Ainda, os autores encontraram que a sarcopenia foi mais prevalente no grupo de idosos que apresentavam quedas (53,5\%), em comparação com o grupo de idosos que não relataram queda $(32,9 \%)$.

A causa da sarcopenia pode ser considerada multifatorial, tendo como gatilhos a diminuição da atividade física; disfunção mitocondrial relacionada à idade; perda de placas terminais do neurônio motor; perda de peso; perda de hormônios anabólicos e aumento de citocinas pró-inflamatórias ${ }^{47}$. A sarcopenia pode impactar negativamente na saúde geral das pessoas idosas, pois se associa ao baixo desempenho físico e declínio funcional, o que pode levar à incapacidade e aumento dos riscos para muitas comorbidades e hospitalização ${ }^{48}$, indo ao encontro dos achados deste estudo, pois o risco de quedas e a pior percepção da capacidade funcional se associaram a sarcopenia, sendo fatores de risco para outras comorbidades, institucionalização e hospitalização. A sarcopenia também foi fator de risco independente para mortalidade (hazard ratio=1,52, IC 95\% 1,06-2,19) . $^{31}$.

Como limitações do estudo tem-se o delineamento do transversal, não permitindo atribuir a causalidade entre as variáveis, além da amostra por conveniência. Além disso o pequeno número de idosos que foram classificados como sarcopênicos pode ser considerado uma limitação do estudo. Sugere-se para os próximos estudos uma abordagem longitudinal, com uma amostra mais numerosa.

\section{CONCLUSÃO}

Idosos com idade avançada, com $\mathrm{IMC}<22 \mathrm{Kg} / \mathrm{m}^{2}$, que eram insuficientemente ativos e que sofreram quedas no ano que antecedeu o estudo foram fatores associados a sarcopenia.

Com este estudo ressalta-se a importância de identificar precocemente os fatores de risco para sarcopenia nas pessoas idosas brasileiras, possibilitando intervenções precoces (grupos de educação em saúde, oficinas de prevenção de quedas e grupos de socialização), podendo 
evitar o surgimento de sarcopenia, assim como evitar os desfechos negativos que a sarcopenia pode acarretar nos idosos. Além disso, estimular as capacidades funcionais dos idosos podem auxiliar como fator protetor da sarcopenia.

\section{REFERÊNCIAS}

1. Cruz-Jentoft AJ, Baeyens JP, Bauer JM, Boirie Y, Cederholm T, Landi F, et al. Sarcopenia: European consensus on definition and diagnosis. Age Ageing. 2010;39(4):412-23. doi: 10.1093/ ageing/afq034.

2. Cruz-Jentoft AJ, Bahat G, Bauer J, Boirie Y, Bruyère O, Cederholm T, et al. Sarcopenia: revised European consensus on definition and diagnosis. Age Ageing. 2019;48(1):16-31. doi: 10.1093/ageing/afy169.

3. Dupuy C, Lauwers-Cances V, Guyonnet S, Gentil C, Van Kan GA, Beauchet $O$, et al. Searching for a relevant definition of sarcopenia: results from the cross-sectional EPIDOS study. J Cachexia Sarcopenia Muscle. 2015;6(2):144-54. doi: 10.1002/ jcsm.12021.

4. Anker SD, Morley JE, von Haehling S. Welcome to the ICD-10 code for sarcopenia. J Cachexia Sarcopenia Muscle 2016;7(5):512-4. doi: 10.1002/jcsm.12147.

5. Shafiee G, Keshtkar A, Soltani A, Ahadi Z, Larijani B, Heshmat R. Prevalence of sarcopenia in the world: a systematic review and meta-analysis of general population studies. J Diabetes Metab Disord. 2017;16(1):21. doi: 10.1186/s40200-017-0302-X.

6. Martone AM, Bianchi L, Abete P, Bellelli G, Bo M, Cherubini A, et al. The incidence of sarcopenia among hospitalized older patients: Results from the Glisten study. J Cachexia Sarcopenia Muscle. 2017:8(6):907-14. doi: 10.1002/jcsm.12224.

7. Buckinx F, Reginster JY, Brunois T, Lenaerts C, Beaudart C, Croisier JL, et al. Prevalence of sarcopenia in a population of nursing home residents according to their frailty status: Results of the SENIOR cohort. J Musculoskelet Neuronal Interact [Internet]. 2017;17(3):209-17. [cited 2021 Jul 28]. Available from: https://bit.ly/3ALhCpf

8. Bianchi L, Ferrucci L, Cherubini A, Maggio M, Bandinelli S, Savino E, et al. The Predictive Value of the EWGSOP Definition of Sarcopenia: Results From the InCHIANTI Study. J Gerontol A Biol Sci Med Sci. 2016;71(2):259-64. doi: 10.1093/gerona/ glv129.

9. Bischoff-Ferrari HA, Orav JE, Kanis JA, Rizzoli R, Schlögl M, Staehelin HB, et al. Comparative performance of current definitions of sarcopenia against the prospective incidence of falls among community-dwelling seniors age 65 and older. Osteoporos Int. 2015;26:2793-802. doi: 10.1007/ s00198-015-3194-y.

10. Schaap LA, van Schoor NM, Lips P, Visser M. Associations of sarcopenia definitions, and their components, with the incidence of recurrent falling and fractures: the longitudinal aging study Amsterdam. J Gerontol A Biol Sci Med Sci. 2018;73(9):1199-204. doi: 10.1093/gerona/gl×245.
11. Malmstrom TK, Miller DK, Simonsick EM, Ferrucci L, Morley JE. SARC-F: a symptom score to predict persons with sarcopenia at risk for poor functional outcomes. J Cachexia Sarcopenia Muscle. 2016;7(1):28-36. doi: 10.1002/jcsm.12048.

12. Bahat G, Ilhan B. Sarcopenia and the cardiometabolic syndrome: a narrative review. Eur Geriatr Med. 2016;7(3):220-23. doi: 10.1016/j.eurger.2015.12.012.

13. Bone AE, Hepgul N, Kon S, Maddocks M. Sarcopenia and frailty in chronic respiratory disease. Chron Respir Dis. 2017;14(1):85-99. doi: $10.1177 / 1479972316679664$.

14. Chang KV, Hsu TH, Wu WT, Huang KC, Han DS. Association between sarcopenia and cognitive impairment: a systematic review and meta-analysis. J Am Med Dir Assoc. 2016;17(12):1164. e7-e15. doi: 10.1016/j.jamda.2016.09.013.

15. Beaudart C, Biver E, Reginster JY, Rizzoli R, Rolland Y, Bautmans I, et al. Validation of the SarQoL(R), a specific health-related quality of life questionnaire for Sarcopenia. J Cachexia Sarcopenia Muscle. 2017;8(2):238-44.

16. Beaudart C, Zaaria M, Pasleau F, Reginster JY, Bruyère O. Health outcomes of sarcopenia: a systematic review and meta-analysis. 2017;12(1):e0169548. doi: 10.1002/jcsm.12149.

17. Vyas L, Nagrale AV. Impact of sarcopenia on quality of life in an elderly population. J Physiother. 2019;1:35-42.

18. Dos Santos L, Cyrino ES, Antunes M, Santos DA, Sardinha LB. Sarcopenia and physical independence in older adults: the independent and synergic role of muscle mass and muscle function. J Cachexia Sarcopenia Muscle. 2017;8(2):245-50. doi: 10.1002/jcsm.12160.

19. Akune T, Muraki S, Oka H, Tanaka S, Kawaguchi H, Tokimura F, et al. Incidence of certified need of care in the long-term care insurance system and its risk factors in the elderly of Japanese population-based cohorts: the ROAD study. Geriatr Gerontol Int. 2014;14(3):695-701. doi:10.1111/ggi.12155.

20. Steffl M, Bohannon RW, Sontakova L, Tufano JJ, Shiells K, Holmerova I. Relationship between sarcopenia and physical activity in older people: a systematic review and meta-analysis. Clin Interv Aging. 2017;12:835-45. doi: 10.2147/CIA.S132940.

21. De Buyser SL, Petrovic M, Taes YE, Toye KRC, Kaufman JM, Lapauw B, et al. Validation of the FNIH sarcopenia criteria and SOF frailty index as predictor of long-term mortality in ambulatory older men. Age Ageing. 2016;45(5):602-8. doi: 10.1093/ageing/afw071.

22. Cawthon PM, Lui LY, Taylor BC, McCulloch CE, Cauley JA, et al. Clinical definitions of sarcopenia and risk of hospitalization in community-dwelling older men: the osteoporotic fractures in men study. J Gerontol A Biol Sci Med Sci. 2017;72(10):1383-89. doi: 10.1093/gerona/glw327.

23. Antunes AC, Araújo DA, Veríssimo MT, Amaral TF. Sarcopenia and hospitalisation costs in older adults: a cross-sectional study. Nutr Diet. 2017;74(1):46-50. doi: 10.1111/1747-0080.12287.

24. Steffl M, Sima J, Shiells K, Holmerova I. The increase in health care costs associated with muscle weakness in older people without long-term illnesses in the Czech Republic: results from the Survey of Health, Ageing and Retirement in Europe (SHARE). Clin Interv Aging. 2017;12:2003-7. doi: 10.2147/CIA. S150826. 
25. Sousa AS, Guerra RS, Fonseca I, Pichel F, Ferreira S, Amaral TF. Financial impact of sarcopenia on hospitalization costs. Eur J Clin Nutr. 2016;70:1046-51.

26. Almeida OP, Almeida SA. Confiabilidade da versão brasileira da Escala de Depressão em Geriatria (GDS) versão reduzida. Arq Neuropsiquiatr. 1999;57(2B):421-6. doi: 10.1038/ejcn.2016.73.

27. Carvalho VA, Caramelli P. Brazilian adaptation of the Addenbrooke's Cognitive Examination-Revised (ACE-R). Dement Neuropsychol. 2007;1(2):212-6. doi: 10.1590/ s1980-57642008dn10200015.

28. Benedetti TB, Mazo GZ, Barros MV. Aplicação do Questionário Internacional de Atividades Físicas para avaliação do nível de atividades físicas de mulheres idosas: validade concorrente e reprodutibilidade teste/reteste. Rev Bras Cien Mov. 2004;12(1):25-33. doi: 10.18511/rbcm.v12i1.538.

29. Ciconelli RM, Ferraz MB, Santos W, Meinão I, Quaresma MR. Tradução para a língua portuguesa e validação do questionário genérico de avaliação de qualidade de vida SF-36 (Brasil SF-36). Rev Bras Reumatol [Internet]. 1999;39(3):143-50. [cited 2021 Jul. 28]. Available from: https://bit.ly/3jWqsJO

30. Alexandre NMC, Gallasch CH, Lima MHM, Rodrigues RCM. A confiabilidade no desenvolvimento e avaliação de instrumentos de medida na área da saúde. Rev Eletr Enferm. 2013;15(3):800-7. doi: 10.5216/ree.v15i3.20776.

31. Alexandre TS, Duarte YAO, Santos JLF, Wong R, Lebrão ML. Prevalence and associated factors of sarcopenia among elderly in Brazil: Findings from the SABE study. J Nutr Health Aging. 2014;18:284-90. doi: 10.1590/1980-549720180009.supl.2.

32. Hulley SB, Cummings SR, Browner WS, Grady DG, Newman TB. Delineando a pesquisa clínica: uma abordagem epidemiológica. 3rd ed. Porto Alegre: Artmed; 2008.

33. Pelegrini A, Mazo GZ, Pinto AA, Benedetti TRB, Silva DAS, Petroski EL. Sarcopenia: prevalence and associated factors among elderly from a Brazilian capital. Fisioter Mov. 2018;31:e003102. doi: 10.1590/1980-5918.031.ao02.

34. Santos CM, Dias JMD, Samora GAR, Perracini MR, Guerra RO, Dias RC. Prevalence of obesity, sarcopenic obesity and associated factors: A FIBRA Network study. Fisioter Mov. 2017;30(Suppl 1):161-9. doi: 10.1590/1980-5918.030.s01.ao16.

35. Sazlina SG, Lee PY, Chan YM, Hamid MSA, Tan NC. The prevalence and factors associated with sarcopenia among community living elderly with type 2 diabetes mellitus in primary care clinics in Malaysia. PLoS One. 2020;15(5):e0233299. doi: 10.1371/ journal.pone.0233299.

36. Dutra T, Pinheiro PA, Carneiro JAO, Coqueiro RS, Fernandes $\mathrm{MH}$. Prevalence and factors associated with sarcopenia in elderly women living in the community. Rev Bras Cineantropom Desempenho Hum. 2015;17(4):460-71. doi: 10.5007/1980-0037.2015v17n4p460.

37. Petermann-Rocha F, Chen M, Gray SR, Ho FK, Pell JP, Celis-Moralesa C. Factors associated with sarcopenia:
A cross-sectional analysis using UK Biobank. Maturitas. 2020;133:60-7. doi: 10.1016/j.maturitas.2020.01.004.

38. Tramontano A, Veronese N, Sergi G, Manzato E, RodriguezHurtado D, Maggi S, et al. Prevalence of sarcopenia and associated factors in the healthy older adults of the Peruvian Andes. Arch Gerontol Geriatr. 2017;68:49-54. doi: 10.1016/j. archger.2016.09.002.

39. Buford TW, Anton SD, Judge AR, Marzetti E, Wohlgemuth SE, Carter CS, et al. Models of accelerated sarcopenia: critical pieces for solving the puzzle of age-related muscle atrophy. Ageing Res Rev. 2010;9(4):369-83. doi: 10.1016/j.arr.2010.04.004.

40. Evans WJ. Skeletal muscle loss: cachexia, sarcopenia, and inactivity. Am J Clin Nutr. 2010;91(4):1123S-7S. doi: 10.3945/ ajcn.2010.28608A.

41. Yang L, Smith L, Hamer M. Gender-specific risk factors for incident sarcopenia: 8-year follow-up of the English longitudinal study of ageing. J Epidemiol Community Health. 2019;73(1):86-8. doi: 10.1136/jech-2018-211258.

42. Yeung SSY, Reijnierse EM, Pham VK, Trappenburg MC, Lim WK, Meskers CGM, et al. Sarcopenia and its association with falls and fractures in older adults: A systematic review and metaanalysis. J Cachexia Sarcopenia Muscle. 2019;10(3):485-500. doi: 10.1002/jcsm.12411.

43. Chen H, Ma J, Liu A, Cui Y, Ma X. The association between sarcopenia and fracture in middle-aged and elderly people: A systematic review and meta-analysis of cohort studies. Injury. 2020;51(4):804-11. doi: 10.1016/j.injury.2020.02.072.

44. Scott D, Hayes A, Sanders KM, Aitken D, Ebeling PR, Jones G. Operational definitions of sarcopenia and their associations with 5-year changes in falls risk in community-dwelling middleaged and older adults. Osteoporos Int. 2014;25(1):187-93. doi: 10.1007/s00198-013-2431-5.

45. Aibar-Almazán A, Martínez-Amat A, Cruz-Díaz D, JiménezGarcía JD, Achalandabaso A, Sánchez-Montesinos I, et al. Sarcopenia and sarcopenic obesity in Spanish communitydwelling middle-aged and older women: Association with balance confidence, fear of falling and fall risk. Maturitas. 2018;107:26-32. doi: 10.1016/j.maturitas.2017.10.001.

46. Lim SK, Beom J, Lee SY, Kim BR, Chun SW, Lim JY, et al. Association between sarcopenia and fall characteristics in older adults with fragility hip fracture. Injury. 2020;51(11):2640-7. doi: 10.1016/j.injury.2020.08.031.

47. Santos ADA, Pinho CPS, Nascimento ACS, Costa ACO, et al. Sarcopenia en pacientes ancianos atendidos ambulatoriamente: prevalencia y factores asociados. Nutr Hosp. 2016;33(2):255-62. doi: 10.20960/nh.100.

48. Mesquita AF, Silva EC, Eickemberg M, Roriz AKC, BarretoMedeiros JM, Ramos LB. Factors associated with sarcopenia in institutionalized elderly. Nutr Hosp. 2017;34(2):345-51. doi: 10.20960/nh.427. 